\title{
EDITORIAL
}

\section{¿Deberíamos preocuparnos por la durabilidad de las válvulas aórticas percutáneas?}

\section{Should we be concerned about the durability of percutaneous aortic valves?}

\author{
Darío Echeverri ${ }^{\mathrm{a}, \mathrm{b}, \mathrm{c}, *}$, Partho P. Sengupta ${ }^{\mathrm{d}, \mathrm{e}}$ y Pedro R. Moreno ${ }^{\mathrm{e}, \mathrm{f}, \mathrm{g}}$
}

\author{
a Servicio de Hemodinamia e Intervencionismo Cardiovascular Fundación Cardioinfantil - Instituto de Cardiología, Bogotá, \\ Colombia \\ ${ }^{\mathrm{b}}$ Laboratorio de Investigación en Función Vascular, Fundación Cardioinfantil - Instituto de Cardiología, Bogotá, Colombia \\ c Facultad de Medicina, Universidad del Rosario, Bogotá, Colombia \\ d Cardiac Ultrasound Research at The Mount Sinai Medical Center, New York, USA \\ e Medicine and Cardiology at The Mount Sinai Medical Center, New York, USA \\ f Icahn School of Medicine at Mount Sinai, New York, USA \\ ' Cardiac Catheterization Laboratory and Interventional Cardiology Mount Sinai Saint Luke's Hospital, New York, USA
}

Recibido el 8 de febrero de 2017; aceptado el 9 de febrero de 2017

Disponible en Internet el 18 de marzo de 2017

El reemplazo de válvulas aórticas transcatéter es una opción de tratamiento excelente para pacientes con estenosis aórtica severa sintomática y riesgo alto o intermedio para cirugía. Con base en evidencia científica sólida en reemplazo de válvulas aórticas transcatéter, obtenida de estudios clínicos aleatorios y con ya cerca de ocho años de experiencia comercial, ¿por qué importaría pensar en la durabilidad de estas válvulas y por qué esta duda acaba de salir a la luz pública?

La durabilidad a largo plazo de las válvulas utilizadas en reemplazo de válvulas aórticas transcatéter, ha sido motivo de interés como respuesta a diferentes factores: El desarrollo continuo de la tecnología reduce los riesgos del procedimiento y mejora la expectativa de vida. Cada vez el reemplazo de válvulas aórticas transcatéter se utiliza con más frecuencia en pacientes jóvenes, aun con patología congénita como la válvula aórtica bivalva, y en aquellas más

\footnotetext{
* Autor para correspondencia.

Correo electrónico: decheverri@cardioinfantil.org (D. Echeverri).
}

complejas incluidas las bioprótesis disfuncionales, con estenosis o regurgitación severa, procedimiento que se conoce como válvula en válvula ${ }^{1,2}$. Sin embargo, pese a la penetración cada vez mayor de la técnica, una gran población con estenosis aórtica severa sintomática, que podría ser potencial candidata a reemplazo de válvulas aórticas transcatéter en un futuro, permanece sin diagnóstico y sin tratamiento.

En la actualidad hay un gran avance en la tecnología, que permite un menor perfil de las válvulas y del sistema de liberación, elaboración con mejores materiales (hidrofílicos), desarrollos en las técnicas de preservación de tejidos ( $3 \underline{a}$ y $4 \stackrel{a}{a}$ generación), diseños recapturables y material más delgado utilizado en la elaboración de la válvula (espesor de folletos de válvulas percutáneas de $\approx 0,25 \mathrm{~mm}$ en comparación con $\approx 0,4 \mathrm{~mm}$ de las valvas de las válvulas quirúrgicas). Estas condiciones ayudan a explicar el mayor uso y la presencia de más de 25 válvulas percutáneas disponibles en todo el mercado mundial ${ }^{3,4}$.

Pese a la excelente evaluación clínica de sobrevida, la evaluación hemodinámica se hizo a largo plazo, hecho que impidió la detección de cambios significativos en pacientes individuales. Estos análisis agrupados enmascaran los 
cambios individuales en gradientes medios y podrían estar sujetos a sesgo de durabilidad. Los hallazgos de inmovilidad de los folletos valvulares o de trombos in-situ, solo son detectables con técnicas cuidadosas de ecocardiograma transesofágico o tomografía cardiaca 4D.

En 2012, el Valve Academic Research Consortium-2 Consensus ${ }^{5}$, elaboró un documento que intentó describir por primera vez los criterios para identificar el deterioro estructural de las válvulas implantadas por vía transcatéter. Se consideraron criterios como gradiente valvular aórtico medio $\geq 20 \mathrm{~mm} \mathrm{Hg}$, área efectiva del orificio valvular $\leq 0,9 \mathrm{~cm}^{2}$, regurgitación valvular protésica moderada o severa con volumen regurgitante $>30 \mathrm{ml}$, fracción regurgitante $>30 \%$ y área efectiva del orificio regurgitante $>0,10 \mathrm{~cm}^{2}$, además de la necesidad de repetir el procedimiento (implante de válvula percutánea o cambio valvular quirúrgico).

En 2013 se publicó uno de los primeros registros clínicos de seguimiento de pacientes sometidos a reemplazo de válvulas aórticas transcatéter ${ }^{6}$, en el que se informaron los resultados a 5 años del implante de válvulas CribierEdwards y Edwards SAPIEN en 88 pacientes en Canadá. Se logró un gradiente medio post-reemplazo de válvulas aórticas transcatéter de $10,0 \pm 4,5 \mathrm{~mm} \mathrm{Hg}$, área valvular aórtica post-reemplazo de válvulas aórticas transcatéter de $1,67 \pm 0,41 \mathrm{~cm}^{2}$ y mortalidad del $0 \%$. Llamó la atención que a 5 años de seguimiento un pequeño porcentaje de pacientes desarrollara regurgitación valvular aórtica moderada o severa. De otro lado, el informe a 5 años del estudio PARTNER 1 en pacientes con estenosis aórtica severa y alto riesgo quirúrgico ${ }^{7}$, así como en pacientes con estenosis aórtica inoperable ${ }^{8}$ no reportó signos de deterioro estructural de las válvulas según la definición del Consensus. En 2015, Barbanti et al. ${ }^{9}$, emitieron los resultados del seguimiento a 5 años de 353 pacientes sometidos a implante de la Core Valve (Medtronic Inc, Minneapolis, Minnesota) de tercera generación en 18 centros de Italia. Describieron fallo tardío severo de la prótesis en 5 casos $(1,4 \%)$, al igual que 2 requerimientos de implante de válvula en válvula por reestenosis sintomática, un caso de endocarditis con regurgitación aórtica severa, uno de deterioro estructural severo de la válvula y uno con fuga paravalvular severa. En 10 pacientes más $(2,8 \%)$ se documentó estenosis aórtica leve tardía con gradiente medio transvalvular de $20-40 \mathrm{~mm} \mathrm{Hg}$.

Por otra parte, en un análisis exhaustivo ${ }^{10}$ hecho para identificar los resultados clínicos y la durabilidad de las válvulas transcatéter con más de dos años de seguimiento en 23 artículos analizados, solo se describió un caso de pseudo-degeneración temprana de la válvula con trombosis a los seis meses y 8 reportes de deterioro estructural de la válvula, 5 de ellos con CoreValve (Medtronic Inc, Minneapolis, Minnesota) y 3 con Edwards SAPIEN. Ningún paciente desarrolló insuficiencia o estenosis aórtica severa ni requirió nueva cirugía o reintervención. Más recientemente, durante el TCT2016 en Washington, se presentaron los resultados del seguimiento a dos años de pacientes después del implante de la válvula Evolut R (Medtronic Inc, Minneapolis, Minnesota). Sesenta pacientes con seguimiento a 30 días, 1 y 2 años, reportaron $0 \%$ de embolización o migración, $0 \%$ de endocarditis, $0 \%$ de disfunción valvular que requiriera nuevos procedimientos y $0 \%$ de trombosis valvular.
Todos estos resultados tenían a la comunidad científica sosegada en términos de durabilidad de la válvula. Sin embargo, en 2016 se identificaron nuevos hallazgos y se definieron nuevos conceptos.

Del trigo et al. ${ }^{11}$, buscaron determinar la incidencia, el momento y los predictores del deterioro hemodinámico de la válvula (DHV) mediante un registro multicéntrico donde incluyeron 1.521 pacientes a quienes se les hizo reemplazo de válvulas aórticas transcatéter; $48 \%$ de ellos, hombres, con edad promedio de $80 \pm 7$ años. Se les realizó ecocardiograma transtorácico al momento del alta, y a los 6 y 12 meses, y luego cada año. Definieron como DHV un aumento del gradiente transvalvular de $\geq 10 \mathrm{~mm} \mathrm{Hg}$. Este estudio permitió identificar los predictores del DHV post-reemplazo de válvulas aórticas transcatéter tales como ausencia de terapia anticoagulante al momento del alta del paciente, procedimiento válvula en válvula, diámetros de la válvula transcatéter $\leq 23 \mathrm{~mm}$ e índice de masa corporal mayor. Este estudio ya pone en el contexto de la práctica clínica la posibilidad de anticoagular a los pacientes por un periodo post-reemplazo de válvulas aórticas transcatéter, principalmente a aquellos con válvula en válvula, diámetros valvulares pequeños y obesos.

Dentro de las causas posibles del deterioro estructural de la válvula percutánea, están la calcificación de la válvula nativa y algunos aspectos técnicos en la preparación de la válvula a implantar. La severidad, extensión y distribución de la calcificación de la válvula nativa puede conducir a algunas situaciones adversas que podrían ayudar a explicar el deterioro estructural de la válvula implantada, tales como la distorsión de la válvula implantada, el favorecimiento de la colocación inapropiada o un implante oblicuo, la expansión incompleta, el aumento de la tensión mecánica en los folletos y la presencia de fugas paravalvulares ${ }^{12-15}$.

Al parecer, los aspectos técnicos pueden influir también en el DEV y de manera importantísima. El tejido utilizado para reemplazo de válvulas aórticas transcatéter es similar al de la válvula quirúrgica (pericardio bovino o porcino) y el tratamiento de anticalcificación utilizado es equivalente al de las válvulas quirúrgicas. Sin embargo, las razones por las que la durabilidad de las válvulas quirúrgicas y percutáneas puede ser diferente, obedecen a la necesidad de reducir el perfil del sistema, utilizando materiales más delgados; adicionalmente, el método de crimping de las valvas puede generar daño tisular microscópico. En los Estados Unidos se han llevado a cabo estudios en modelos computacionales de fatiga de tejidos ${ }^{4}$, para comparar la durabilidad del reemplazo de válvulas aórticas transcatéter vs. las válvulas bioprotésicas quirúrgicas en condiciones óptimas, y se ha obervado que la durabilidad de los dispositivos de reemplazo de válvulas aórticas transcatéter se reduce en comparación con las válvulas quirúrgicas $a \approx 7,8$ años; se espera que se reduzca aún más la durabilidad en condiciones no óptimas (anatomía elíptica o implante subexpandido). Así mismo, el crimping, el implante y la dilatación con balón de válvulas percutáneas puede causar diversos grados de lesiones, deformaciones y roturas en la estructura de colágeno de los folletos de pericardio de las válvulas implantadas, lo cual promueve la adhesión y captación de plaquetas y exacerba la trombogenicidad ayudando al razonamiento creciente que sea cuestionable la durabilidad a largo plazo de 


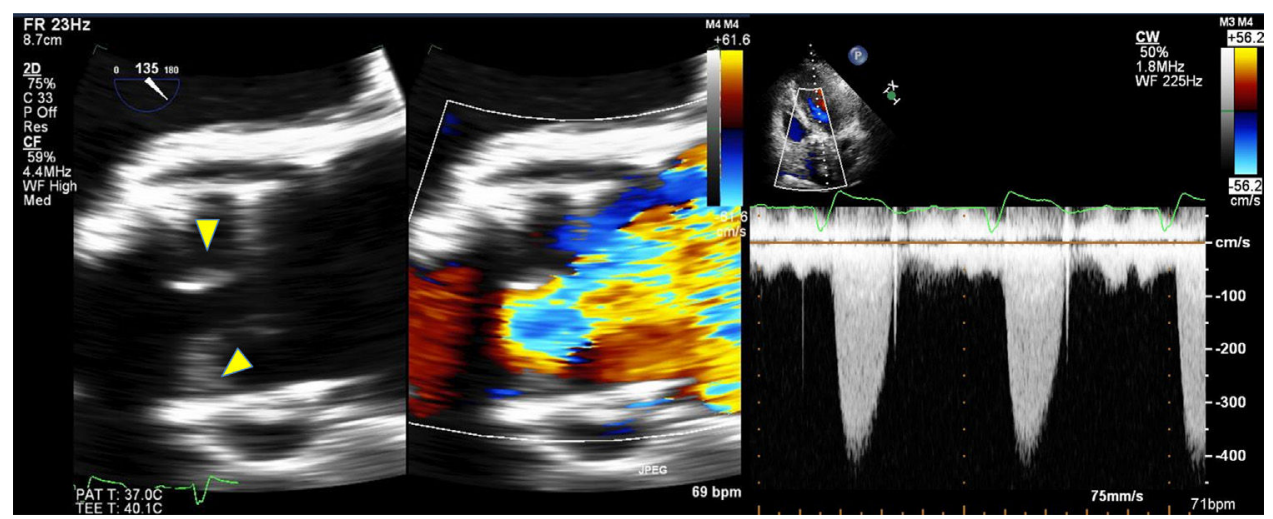

Figura 1 Engrosamiento y restricción en la movilidad de los folletos valvulares post-reemplazo de válvulas aórticas transcatéter (Edwards Lifesciences, Irvine, California) con estenosis aórtica severa.

la biofuncionalidad de las válvulas implantadas en forma percutánea ${ }^{16}$.

En general, las válvulas bioprotésicas son menos trombogénicas que las mecánicas. No obstante, entre los riesgos del DEV descrito anteriormente se puede generar disminución de la movilidad de folletos y trombosis consecuente. Según estudios previos en válvulas quirúrgicas, se espera una incidencia de trombosis valvular cercana al $1 \%$. Recientemente, gracias a métodos de seguimiento con imágenes más sofisticados, como TAC-multidectectora de alta resolución, se ha encontrado una incidencia de trombosis en reemplazo de válvulas aórticas transcatéter hasta del $7 \%$ a 3 meses, lo cual podría tener implicaciones relevantes. Hansson et al. ${ }^{17}$, evaluaron la incidencia, los predictores y las implicaciones clínicas de la trombosis de reemplazo de válvulas aórticas transcatéter mediante TAC multidetectora en 460 pacientes consecutivos sometidos a reemplazo de válvulas aórticas transcatéter Edwards Sapien XT o Sapien 3 (Edwards Lifesciences, Irvine, California). En 405 (88\%) pacientes que se sometieron a TAC y ecocardiograma transesofágico (ETE) 1-3 m post-reemplazo de válvulas aórticas transcatéter, se demostró engrosamiento con hipoatenuación de los folletos sugestivos de trombosis aun sin signos de obstrucción en ETT (GM: $9 \mathrm{~mm} \mathrm{Hg}$, área del orificio valvular: $1,6 \mathrm{~cm}^{2}$ ) y resolución completa del trombo después de tres meses de tratamiento con warfarina y aspirina. La TAC multidetectora de alta resolución, permitió identificar trombosis de la válvula aórtica transcatéter en 28 de 405 pacientes (7\%), 23 pacientes con trombosis subclínica y 5 (18\%) con trombosis obstructiva. La trombosis de la válvula aórtica percutánea fue igual en las diferentes generaciones de válvulas ( $8 \%$ vs. $6 \%, p=0,42)$. La trombosis de la válvula aórtica transcatéter en quienes no recibieron warfarina fue mayor que en aquellos que sí la recibieron (10,7\% vs. $1,8 \%$, RR: 6,09; IC 95\%: 1,86 - 19,84). Con base en estos hallazgos coinciden algunos factores de DEV y de trombosis de las válvulas aórticas transcatéter, tales como la no anticoagulación con warfarina, el aumento del gradiente transvalvular, el incremento del espesor de las cúspides, la movilidad anómala de las cúspides y el tamaño de la válvula empleada (necesita confirmación) (fig. 1).

En conclusión, datos clínicos sólidos a largo plazo de reemplazo de válvulas aórticas transcatéter sólo están disponibles para un máximo de 5 años. La experiencia con válvulas aórticas quirúrgicas indica que se requieren más de 10 años de seguimiento en pacientes de edad avanzada para analizar la durabilidad de la válvula. Existe una asociación inversa entre la edad y el deterioro de la válvula estructural. Por tanto, se precisa cautela al considerar el reemplazo de válvulas aórticas transcatéter en situaciones de riesgo bajo, intermedio y en pacientes jóvenes. Se desconoce aún la incidencia de DEV y trombosis. Es importante la monitorización del gradiente transvalvular (ETE); según lo descrito anteriormente, si hay aumento de $>10 \mathrm{~mm} \mathrm{Hg}$, se recomiendan técnicas de imagen más sofisticadas (valor por ser validado) y considerar la anticoagulación oral. Todavía no está claro si la terapia antiplaquetaria sola es suficiente o se necesitan antagonistas de la vitamina $\mathrm{K}$, o si deba añadirse un nuevo anticoagulante oral durante los primeros 3 a 6 meses. Para saber si la terapia con anticoagulación debe considerarse en los primeros tres meses en pacientes con mayor riesgo de trombosis valvular, incluidos aquellos que han tenido un procedimiento válvula en válvula, están pendientes los resultados de estudios en curso: GALILEO (the Global Study Comparing a rivAroxaban-based Anti-thrombotic Strategy to an antipLatelet-based Strategy After Transcatheter aortlc valve rEplacement to Optimize Clinical Outcomes), evalua rivaroxaban versus DAPT, CLOE (Clopidogrel to Lower advErse ischemic events after transcatheter aortic valve) (aspirin vs. dual antiplatelet therapy) y ATLANTIS(AntiThrombotic Strategy After TransAortic Valve Implantation for Aortic Stenosis) (apixaban $5 \mathrm{mg} 2$ vs. dual antiplatelet therapy or vitamin $\mathrm{K}$ antagonist).

\section{Bibliografía}

1. Walther T, Hamm CW, Schuler G, Berkowitsch A, Kötting J, Mangner N, et al., GARY Executive Board. Perioperative results and complications in 15,964 transcatheter aortic valve replacements: prospective data from the GARY registry. JACC. 2015;65:2173-80.

2. Holmes DR Jr, Brennan JM, Rumsfeld JS, Dai D, O’Brien SM, Vemulapalli S, et al., STS/ACC TVT Registry. Clinical outcomes at 1 year following transcatheter aortic valve replacement. JAMA. 2015;313:1019-28.

3. Thubrikar MJ, Deck JD, Aouad J, Nolan SP. Role of mechanical stress in calcification of aortic bioprosthetic valves. J Thorac Cardiovasc Surg. 1983;86:115-25. 
4. Martin C, Sun W. Comparison of transcatheter aortic valve and surgical bioprosthetic valve durability: A fatigue simulation study. J Biomech. 2015;48:3026-34.

5. Kappetein AP, Head SJ, Généreux P, Piazza N, van Mieghem NM, Blackstone EH, et al. Valve Academic Research Consortium-2. Updated standardized endpoint definitions for transcatheter aortic valve implantation: the Valve Academic Research Consortium-2 consensus document. Eurolntervention. 2012;8:782-95.

6. Toggweiler S, Humphries KH, Lee M, Binder RK, Moss RR, Freeman $\mathrm{M}$, et al. 5-year outcome after transcatheter aortic valve implantation. J Am Coll Cardiol. 2013;61:413-9.

7. Mack MJ, Leon MB, Smith CR, Miller DC, Moses JW, Tuzcu EM, et al., for the PARTNER 1 trial investigators. 5-year outcomes of transcatheter aortic valve replacement or surgical aortic valve replacement for high surgical risk patients with aortic stenosis (PARTNER 1): a randomised controlled trial. Lancet. 2015;385:2477-84.

8. Kapadia SR, Leon MB, Makkar RR, Tuzcu EM, Svensson LG, Kodali $\mathrm{S}$, et al., for the PARTNER trial investigators. 5-year outcomes of transcatheter aortic valve replacement compared with standard treatment for patients with inoperable aortic stenosis (PARTNER 1): a randomised controlled trial. Lancet. 2015;385:2485-91.

9. Barbanti M, Petronio AS, Ettori F, Latib A, Bedogni F, De Marco $F$, et al. 5-year outcomes after transcatheter aortic valve implantation with corevalve prosthesis. JACC Cardiovasc Interv. 2015;8:1084-91.

10. Sulzenko J, Tousek P, Kocka V, Widimsky P. Transcatheter aortic valve implantation: long-term clinical outcome and valve durability. Expert Rev Med Devices. 2015:1-7.

11. Del Trigo M, Muñoz-Garcia AJ, Wijeysundera HC, NombelaFranco L, Cheema AN, Gutierrez E, et al. Incidence, timing, and predictors of valve hemodynamic deterioration after transcatheter aortic valve replacement: multicenter registry. J Am Coll Cardiol. 2016;67:644-55.

12. Hayashida K, Lefèvre T, Chevalier B, Hovasse T, Romano M, Garot P, et al. Impact of post-procedural aortic regurgitation on mortality after transcatheter aortic valve implantation. JACC Cardiovasc. Interv. 2012;5:1247-56.

13. Kodali SK, Williams MR, Smith CR, Svensson LG, Webb JG, Makkar RR, et al., PARTNER Trial Investigators. Two-year outcomes after transcatheter or surgical aortic-valve replacement. N Engl J Med. 2012;366:1686-95.

14. Tamburino C, Capodanno D, Ramondo A, Petronio AS, Ettori F, Santoro G, et al. Incidence and predictors of early and late mortality after transcatheter aortic valve implantation in 663 patients with severe aortic stenosis. Circulation. 2011;123:299-308

15. Abdel-Wahab M, Zahn R, Horack M, Gerckens U, Schuler G, Sievert $\mathrm{H}$, et al., German transcatheter aortic valve interventions registry investigators. Aortic regurgitation after transcatheter aortic valve implantation: incidence and early outcome. Results from the German transcatheter aortic valve interventions registry. Heart. 2011;97:899-906.

16. Bourget JM, Zegdi R, Lin J, Wawryko P, Merhi Y, Convelbo C, et al. Correlation between structural changes and acute thrombogenicity in transcatheter pericardium valves after crimping and balloon deployment. J Morpho. 2016;282:1-14.

17. Hansson NC, Grove EL, Andersen HR, Leipsic J, Mathiassen ON, Jensen JM, et al. Transcatheter aortic valve thrombosis: incidence, predisposing factors, and clinical implications. J Am Coll Cardiol. 2016;68:2059-69. 